\title{
Initiation of hot coronal loop oscillations: Spectral features
}

\author{
T. J. Wang, S. K. Solanki, D. E. Innes, and W. Curdt
}

\author{
Max-Planck Institut für Sonnensystemforschung, 37191 Katlenburg-Lindau, Germany ${ }^{\star}$ \\ e-mail: wangtj@linmpi.mpg.de
}

Received 11 January 2005 / Accepted 14 February 2005

\begin{abstract}
We explore the excitation of hot loop oscillations observed with the SUMER spectrograph on SOHO by analysing Fe XIX and Fe XXI spectral line profiles in the initial phase of the events. We investigate all 54 Doppler shift oscillations in 27 flare-like events, whose physical parameters have been measured so far. In nearly $50 \%$ of the cases, the spectral evolution reveals the presence of two spectral components, one of them almost undisturbed, the other highly shifted. We find that the shifted component reaches maximum Doppler shift (on the order of $100-300 \mathrm{~km} \mathrm{~s}^{-1}$ ) and peak intensity almost simultaneously. The velocity amplitude of the shifted component has no correlation with the oscillation amplitudes. These features imply that in these events the initial shifts are not caused by the locally oscillating plasma (or waves), but most likely by a pulse of hot plasma travelling along the loop through the slit position. This interpretation is also supported by several examples showing that standing slow mode waves are set up immediately after the initial line shift pulse (standing slow mode waves are inferred from the 1/4-period phase relationship between the velocity and intensity oscillations). We re-measure the physical parameters of the 54 Doppler oscillations by fitting the time profiles excluding the first peak, and find that the periods are almost unchanged, damping times are shorter by $5 \%$, and amplitudes are smaller by $37 \%$ than measured when the first peak is included. We also measure the velocity of the net (background) flow during the oscillations, which is found to be nearly zero. Our result of initial hot flows supports the model of single footpoint (asymmetric) excitation, but contradicts chromospheric evaporation as the trigger.
\end{abstract}

Key words. Sun: corona - Sun: flares - Sun: oscillations - Sun: UV radiation

\section{Introduction}

MHD waves and oscillations have been discussed for several decades in connection with solar coronal heating and solar wind acceleration. Recent observations of coronal waves and oscillations in combination with theoretical models are leading to diagnostic tools for determining otherwise inaccessible parameters of the coronal plasma (e.g. Roberts et al. 1984; Roberts 2000; Nakariakov \& Ofman 2001; Roberts \& Nakariakov 2003). Imaging observations with SOHO and TRACE in the EUV spectral range have detected fast global kink-mode oscillations in cool $(T \approx 1 \mathrm{MK})$ coronal loops, standing slow mode oscillations in hot $(T \approx 6-8 \mathrm{MK})$ coronal loops, and propagating slow magneto-acoustic waves in polar plumes and long fan-like coronal loops (see the reviews by Aschwanden 2003; Wang 2004).

Strongly damped Doppler-shift oscillations in hot flare lines were first revealed by the SUMER spectrometer on SOHO (e.g. Wang et al. 2002, 2003b, and references therein), and have been interpreted in terms of standing slow waves (Ofman \& Wang 2002; Wang et al. 2003a). These oscillations have periods in the range of 7 to $31 \mathrm{~min}$ and decay within a couple of periods. For all cases, the initially rapid increase of the line intensity and initial large Doppler broadenings indicate that the

^ Previously known as MPI für Aeronomie. oscillations are excited impulsively. Most SUMER oscillation events happened without associated GOES flares, although the initiation of some events was associated with the brightening of a footpoint of the oscillating loop (Wang et al. 2003b), suggesting that the trigger of the oscillations could be micro- or subflares. So far the excitation of these oscillations has not yet been investigated in detail. In particular, the information present in the spectral line profiles has not been fully exploited. It can offer another perspective on dynamic processes in coronal loops and allow the excitation mechanism of the oscillations to be explored. In this study, we analyze the evolution of Fe XIX and Fe XXI spectral profiles in the initial phase of the 54 Doppler shift oscillations previously reported by Wang et al. (2003b) (hereinafter Paper I). The observations are described in Sect. 2, three selected examples are analysed in Sect. 3, statistical properties are presented in Sect. 4, and the excitation of the oscillations is discussed in Sect. 5. Finally, the obtained results are summarized in Sect. 6.

\section{Observations}

Out of the 27 oscillation events reported in Paper I, we have selected three examples for a detailed analysis. They were observed in an Fe XIX line on the 11th, 16th, and 17th of April 2002, and are listed as No. 11, 18, and 20 in 
Table 1 of Paper I. During the observing sequence, spectra of the Si III $1113 \AA$ (0.03-0.06 MK), Ca X $557 \AA$ (2nd order, $0.7 \mathrm{MK})$, and Fe XIX $1118 \AA$ (6.3 MK) lines were recorded with a 50 s cadence using the wide slit $\left(300^{\prime \prime} \times 4^{\prime \prime}\right)$ placed at a fixed position above the solar limb. For each line, a $2.2 \AA$ wide window was transmitted. First, a single Gaussian was fitted to the line profiles to obtain a Doppler shift time series at each spatial pixel. Since, at the early stages of many events the line profiles are complex and a single Gaussian is not an adequate representation we have later carried out a more thorough analysis of the complex profiles involving fits with multiple Gaussians. Note that Doppler shifts of the Fe XIX line reported in Paper I were determined relative to a reference wavelength which is taken as the line center position averaged over the entire data set, since any background flow was not important for oscillation measurements. In order to investigate background flows associated with the oscillations, we now determine the Doppler shifts of the Fe XIX line relative to the nearby Si III line center position. Si III emission is normally only seen as scattered light from the disk and therefore net Doppler flows are not expected.

\section{Case study - evidence for initial ejected flows}

\subsection{April 2002}

This oscillation event was seen by SUMER as two brightenings (denoted A and B in Fig. 1) associated with large initial red shifts in the Fe XIX line. According to Fig. 1b brightening B showed a line shift several minutes later than A. These two brightenings were located near the intersections of the slit with a coronal loop seen in TRACE. SOHO/EIT images from previous days show that this loop system belonged to AR 9893 where most loop systems were oriented clockwise relative to the solar equator. This implies that the northern footpoint (F1) is nearer to the observer, in agreement with the stronger brightness at F1 than at F2 (Fig. 1a). Although the TRACE image shows gas that is at much lower temperature than Fe XIX, we expect from Fig. 1 that the hot loop sampled by SUMER runs closely parallel to the TRACE loop. This is reasonable if they belong to the same loop system. Using the circular loop model described in Paper I, we obtain the azimuth angle of the loop baseline to the EW direction $\alpha=30^{\circ}$, the inclination angle of the loop plane northward (away from the observer) to the vertical $\theta=30^{\circ}$, and the loop length $L=173 \mathrm{Mm}$. We can also determine angles to the line-of-sight of the vectors tangential to the loop (defined along the loop pointing toward the footpoint F2) close to cuts $\mathrm{A}$ and $\mathrm{B}: \gamma_{\mathrm{A}}=151^{\circ}$ and $\gamma_{\mathrm{B}}=116^{\circ}$. Therefore, if a hot plasma flow ejected near the footpoint F1 moves upward along the loop it will cross the slit position twice and an initial flow will first be observed as a red shift in A and then with a time delay in B, with the velocity in A being larger. This signature is seen in the line profiles, as illustrated in Fig. 1b.

Another loop system seen in the TRACE image near F2 (position C) rose gradually up to the SUMER slit at the position of strong emission in Fe XIX. When these loops reached the slit, SUMER measured a strong, compact brightening in $\mathrm{Ca} X$

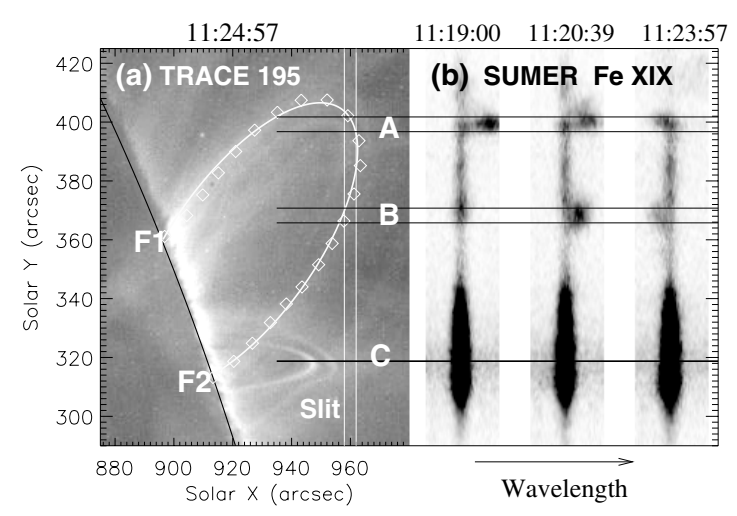

Fig. 1. Observations of the initial stages of a hot loop oscillation event on 17 April 2002. a) TRACE image. A coronal loop (outlined with diamonds) is fit with a circular model (white curve). The SUMER slit is indicated by two vertical lines, and positions of two cuts (denoted A and B) are marked. b) Spectra along the slit in the Fe XIX line at three times during the event onset phase.

(a) Line-of-sight Doppler shift
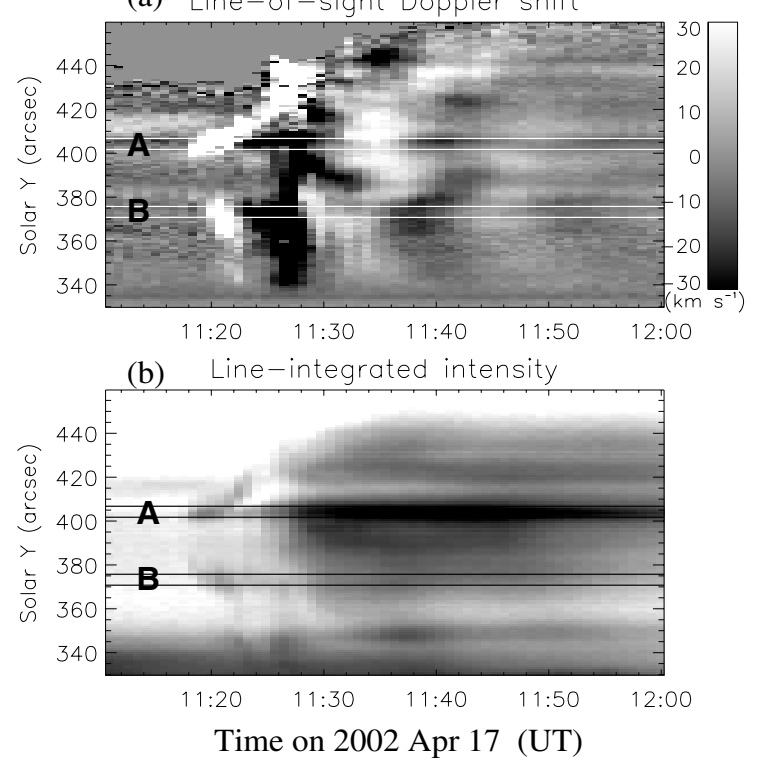

Fig. 2. a) Doppler shift time series of the Fe XIX line at a fixed slit position. Redshift is represented in pale, and blueshift in dark tint. The labels A and B mark sections with widths of 6 pixels along the slit at the same positions as shown in Fig. 1. b) Line-integrated intensity time series with brightness on a logarithmic scale.

and Si III, indicative of the cooling of a hot flaring loop system. The good coincidence in position of the loop's apex and the CaX brightening shows that the SUMER slit and the TRACE images are coaligned to an accuracy of better than $5^{\prime \prime}$ in the solar $y$ direction.

The evolution of (line-of-sight) Doppler shift and intensity of Fe XIX emission along the slit are shown in Fig. 2. In Paper I we report the best fit damped sine function to the time profiles for A and B. The oscillation periods are 13.2 and $13.1 \mathrm{~min}$, and the decay times are 8.4 and $9.7 \mathrm{~min}$. The similarity of periods and phases of the oscillations at points A and B strongly suggests that they occur in the same oscillating loop. For the intensity at $\mathrm{A}$ and $\mathrm{B}$, we find that the initial redshifts 


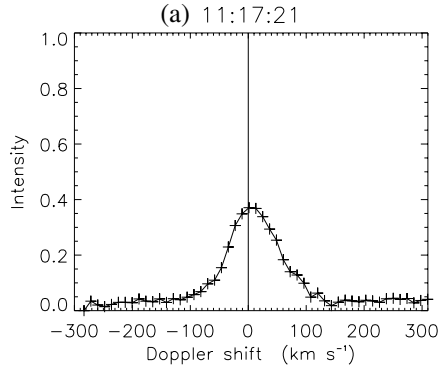

(d) $11: 22: 18$

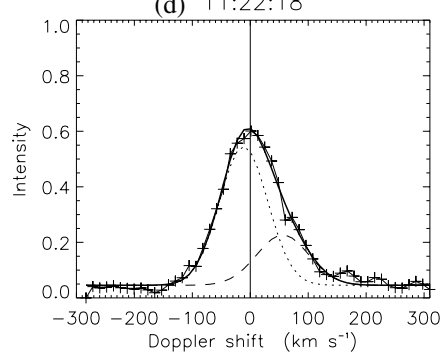

(b) 11:19:00

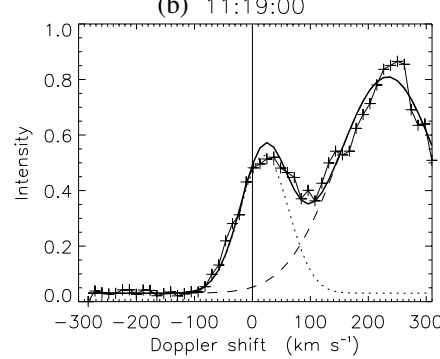

(e) $11: 23: 57$

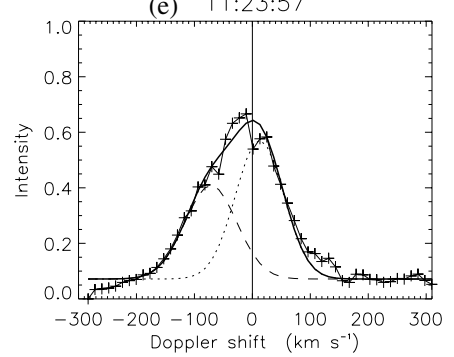

(c) $11: 20: 39$

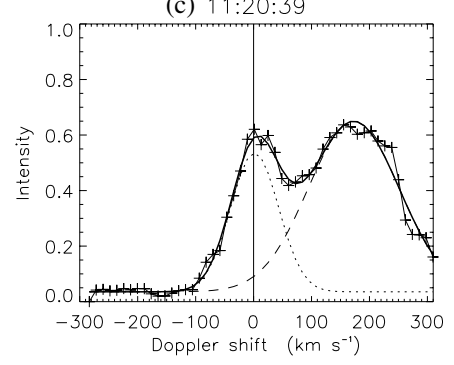

(f) $11: 25: 36$

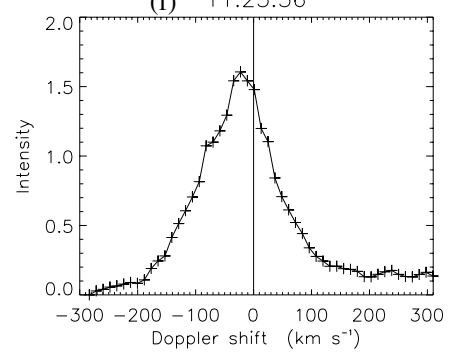

Fig. 3. Evolution of Fe XIX line profiles (crosses) for cut A. In panels b)-e), the thick solid curve is a two-Gaussian fit, with one component, given by the dotted line, of fixed amplitude and line width. The spectral intensity is in units of counts $\mathrm{s}^{-1}$ spatial-pixel $^{-1}$ spectral-pixel $^{-1}$.

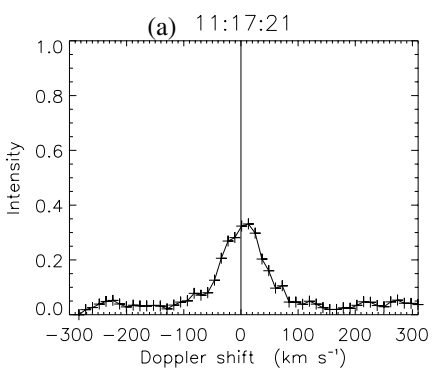

(d) $11: 22: 18$

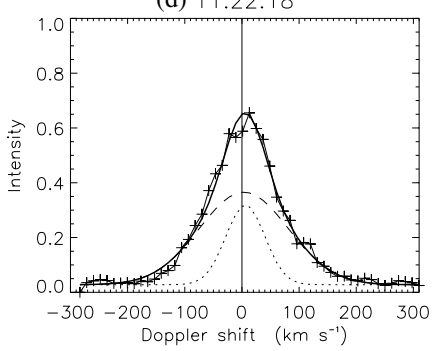

(b) 11:19:00

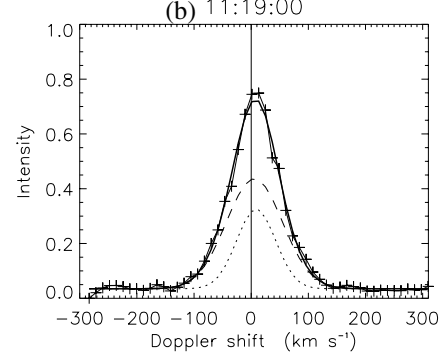

(e) $11: 23: 57$

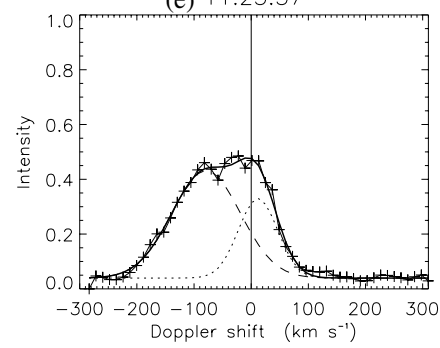

(c) 11:20:39

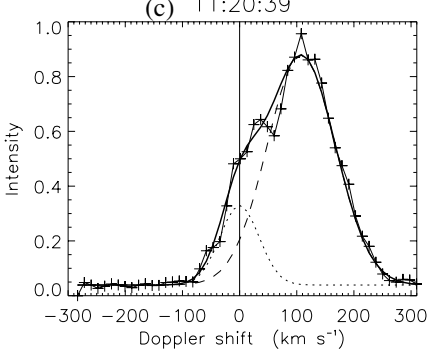

(f) $11: 25: 36$

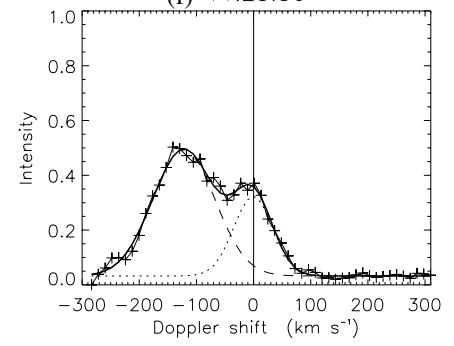

Fig. 4. Same as Fig. 3 but for cut B.

correspond to rapid brightenings from 11:18 to 11:22, while during the following blueshifts the intensity slightly decreases. The intensity of A started to increase again at 11:25, and the intensity of B at 11:29, respectively. Note that a flare occurred behind the limb, which is seen as a GOES brightening at 11:24 (Wang \& Solanki 2004). In SUMER the effect of the flare is seen at 11:26. SUMER frames after this time are affected by this flare and are not considered further.

We now diagnose the evolution of the Fe XIX line profiles during the initial phase (i.e. before the strong emission at 11:30). Figure 3 clearly shows that the line profiles at A consist of two components; a stationary component with a weak intensity increase and a small Doppler shift, and a high Doppler velocity component with an impulsively increasing intensity and a large line width. Assuming that the stationary component has a fixed amplitude and line width, a two-Gaussian function provides good fits to the line profiles observed between 11:18 and 11:24. By keeping one component fixed the stability of the fit is enhanced. A component with a high Doppler shift of $96 \mathrm{~km} \mathrm{~s}^{-1}$ appeared at the red wing at 11:18:10, and reached its peak intensity and maximum shift of $234 \mathrm{~km} \mathrm{~s}^{-1}$ at 11:19:49 before decaying (Figs. 3b-d). A new component appeared in the blue wing at $67 \mathrm{~km} \mathrm{~s}^{-1}$ a few minutes later (Fig. 3e). Figure 4 shows that a redshifted component at position B appeared about 2 min later than at A. This component has a maximum Doppler velocity of $108 \mathrm{~km} \mathrm{~s}^{-1}$ at 11:20:39 (Fig. 4c). Later, a new blueshifted component emerged almost simultaneously with that at A (Fig. 4e). This component reached its maximum Doppler velocity of $136 \mathrm{~km} \mathrm{~s}^{-1}$ at 11:26:25.

Based on the assumption that either the oscillating loop is the TRACE loop or runs nearly parallel to it (which is reasonable if they belong to the same loop system), we now 

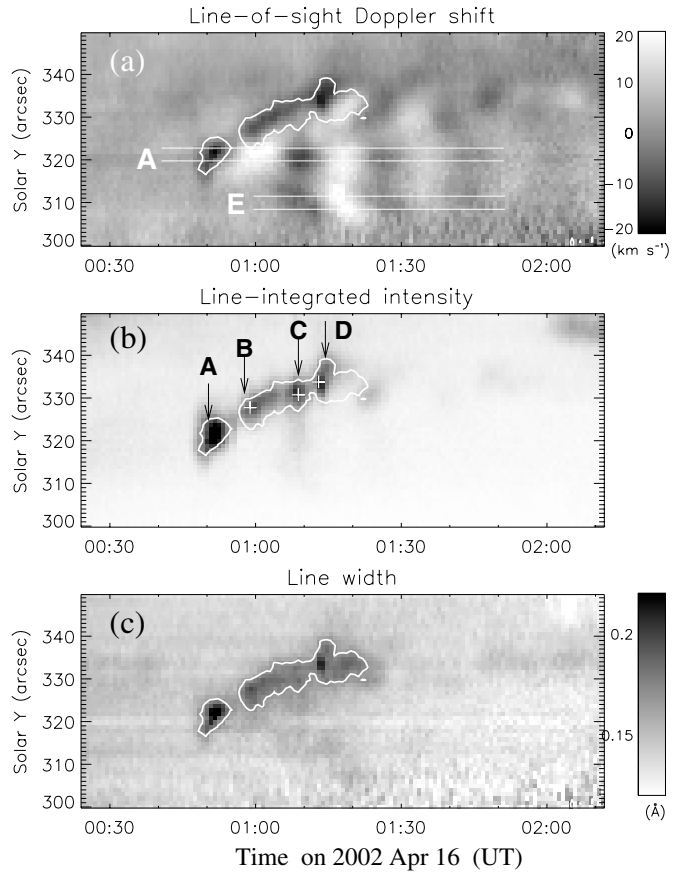

Fig. 5. a) Map of velocity along the slit vs. time for the $1118 \AA$ Fe XIX line. The label A marks the section, with a width of 4 pixels along the slit, at which the disturbance is first seen. The label $\mathrm{E}$ marks an oscillation with a time lag of one period relative to the oscillation at A. b) Line intensity time series integrated at the blue wing from a Doppler shift of $100 \mathrm{~km} \mathrm{~s}^{-1}$ to $160 \mathrm{~km} \mathrm{~s}^{-1}$. The crosses labelled B-D mark the positions where the line profiles are shown in Fig. 8. c) Line width (measured Gaussian width) time series. The overlaid contours outline areas of line width above $0.17 \AA$.

discuss the transit time of the flow along the loop and compare it with the observations. From the 3-D geometry, we obtain $33 \mathrm{Mm}$ for the length of the arch section from A to B. For cut A, we measure the maximum red Doppler velocity of $V_{z}=234 \mathrm{~km} \mathrm{~s}^{-1}$ and calculate a flow speed of $V_{\mathrm{a}}=267 \mathrm{~km} \mathrm{~s}^{-1}$ by $V_{\mathrm{a}}=V_{z} / \cos \gamma_{\mathrm{A}}$. For cut $\mathrm{B}$ the similarly calculated flow speed is $V_{\mathrm{b}}=246 \mathrm{~km} \mathrm{~s}^{-1}$. Taking the mean value of the flow speeds at $\mathrm{A}$ and $\mathrm{B}$, we estimate a transit time of the flow from $\mathrm{A}$ to $\mathrm{B}$ of $124 \mathrm{~s}$, which agrees with the observed time lag of $99 \pm 50 \mathrm{~s}$ within the error bars (which reflect the cadence of the observations). This estimate supports the idea of a pulse of gas flowing in the loop. The near simultaneous appearance of the blueshift at both locations A and B after 11:23 is consistent with the subsequent setup of a standing slow mode wave. If this interpretation is correct, it implies that the standing wave is set up after only a single bounce of the initial disturbance.

\subsection{April 2002}

In this example, Wang et al. (2003a) found strong evidence for the presence of slow mode standing waves in the oscillating loop, indicated by the characteristic phase shift of a quarter period between Doppler and intensity oscillations, which was established after the initial Doppler shift pulse. Evolution of Doppler shift, blue wing intensity and line width of the Fe XIX line along the slit are shown in Fig. 5. The
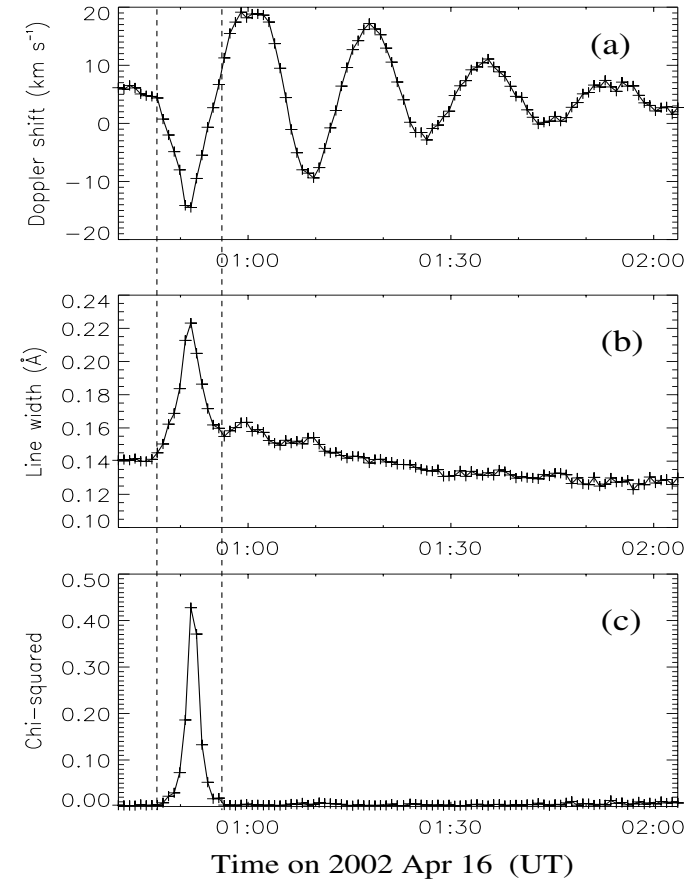

Fig. 6. a) Time profile of Doppler shifts for cut A shown in Fig. 5a, averaged along the slit. b) Corresponding time profile of line widths. c) Corresponding time profile of $\chi^{2}$ for a single Gaussian fit to the line profiles, normalized by line intensity.

event starts with a feature propagating northward along the slit with a speed of about $8 \mathrm{~km} \mathrm{~s}^{-1}$. We find that this propagating feature is actually composed of a sequence of blueshifted brightenings (labelled A, B, C, and D in Fig. 5b), which occurred at 00:51:32, 00:58:58, 01:08:52, and 01:12:59, respectively. These blueshifted brightenings are associated with large Doppler broadenings (Fig. 5c).

We have selected brightening A for a more detailed analysis. Figure 6 shows that its initial blue shift clearly coincides with a strong pulse in line width and $\chi^{2}$ (between two vertical dashed lines in all frames). The large values of $\chi^{2}$ imply that a single Gaussian is a poor fit to the initial line profiles. Figure 7 displays the evolution of the Fe XIX line profiles at A during the initial phase. We find that a new component emerged in the blue wing of the background component after 00:47. The shifted component reached its maximum velocity of $110 \mathrm{~km} \mathrm{~s}^{-1}$ at 00:51:32, as determined from a double Gaussian fit. The background component started to oscillate after 00:56 when the shifted component had almost completely decayed. The evolution of line profiles at B, C and D is similar to that at A, also indicating the occurrence of shifted components before the oscillations. The maximum shifts at $\mathrm{B}, \mathrm{C}$, and $\mathrm{D}$ are determined to be 87,112 , and $119 \mathrm{~km} \mathrm{~s}^{-1}$, respectively (Fig. 8).

The evolution of Doppler shift, line intensity and line width at A measured by single Gaussian fits and double Gaussian fits is illustrated in Fig. 9. We find that the Doppler velocity of the initial blueshifted component varies in a narrow range of $80-110 \mathrm{~km} \mathrm{~s}^{-1}$ and is much larger than the velocity amplitude measured by single Gaussian fits. The shifted component exhibits an impulsive intensity variation and strong line broadening, in distinct contrast to the behavior of the background 

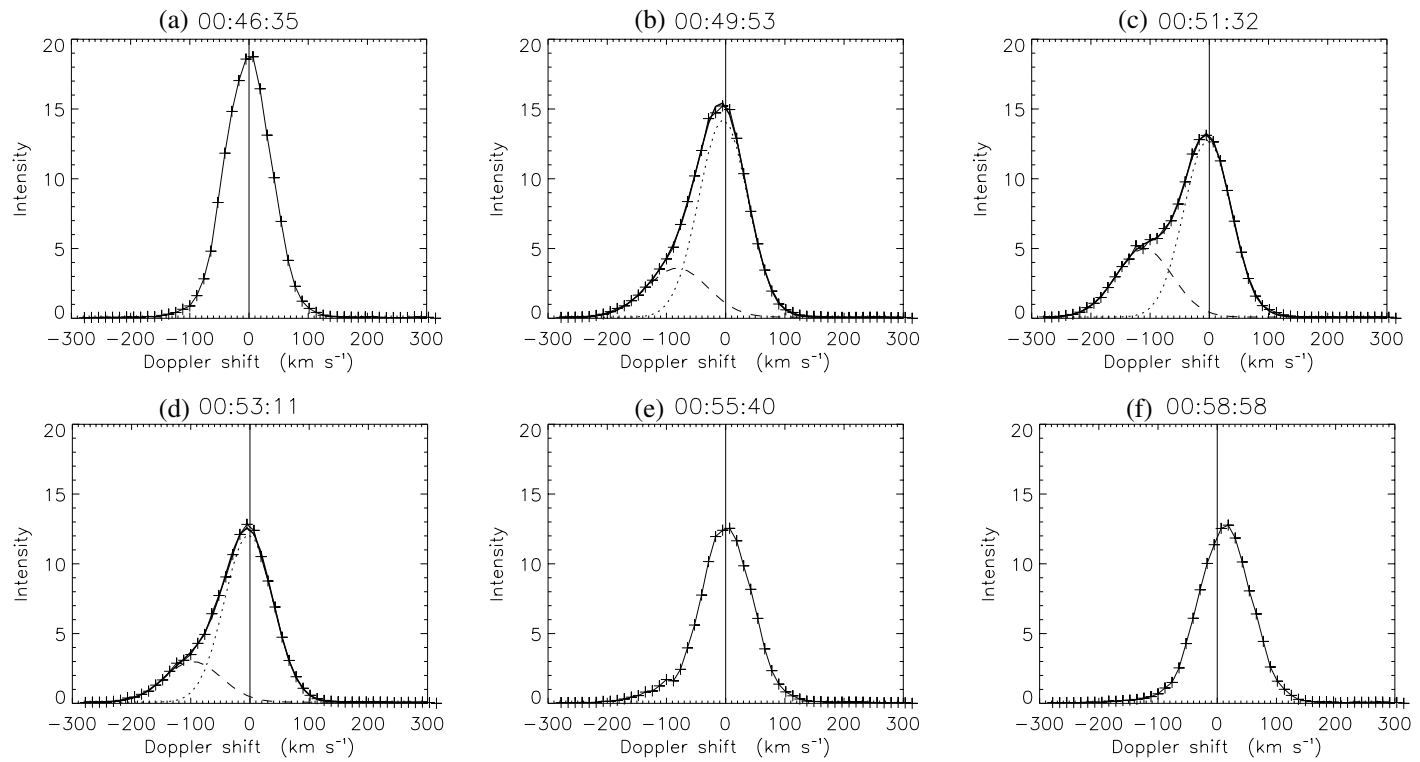

Fig. 7. Evolution of the Fe XIX line profiles (crosses) for cut A. In panels b)-d), the thick solid curve is a two-Gaussian fit, with the two components represented by the dotted and the dashed line, respectively. The spectral radiance is in instrumental units.
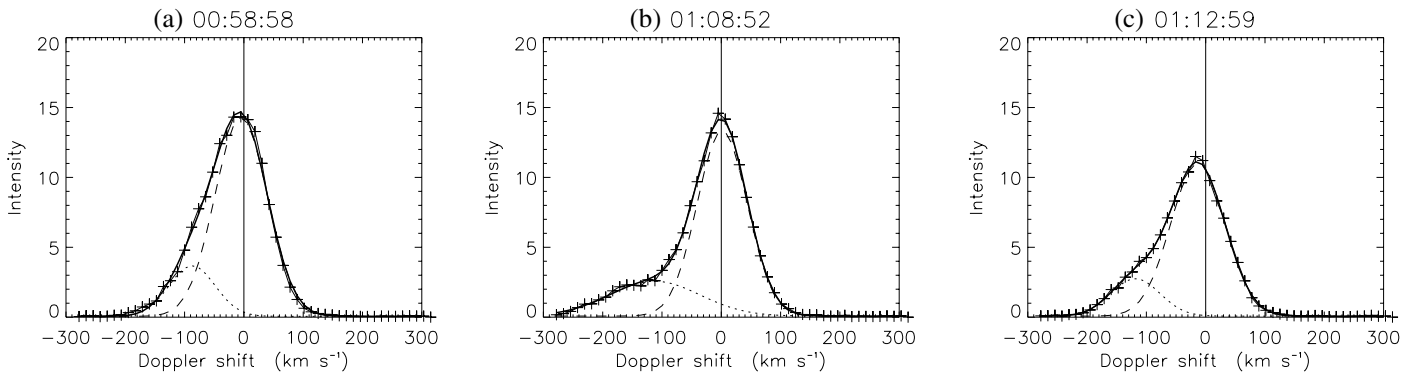

Fig. 8. Fe XIX line profiles for brightenings a) B; b) C; and c) D, at positions marked in Fig. 5b. The various curves have the same meanings as in Fig. 7.

component. This observation indicates that the initial pulse of shifted emission is not produced by the oscillations of the coronal plasma in the loop, and suggests a different explanation. An impulsively ejected hot flow could cause this shifted component which then excites the oscillations of the background component emitted from the coronal plasma in loop A. According to Fig. 9 the background component starts to oscillate only after the impulsive, blue shifted component has decayed or moved out of the slit. At this point also the $\pi / 2$ phase shift between velocity and intensity oscillations is established. The other three blueshifted brightenings along the initial propagating feature could be caused by a series of plasma ejections in three neighboring loops $\mathrm{B}, \mathrm{C}$ and $\mathrm{D}$.

For the Doppler oscillation at position E (Fig. 5), however, the evolution of initial line profiles is different. The oscillation starts as a simple blueshift of the background component with a weak line broadening. We will discuss excitations of this type in Sect. 4.

\subsection{April 2002}

In Paper I, we found a phase propagation in some Doppler oscillations, and suggested two interpretations: the excitation of the oscillations in a loop with fine structure (or in a loop system), or propagating waves. Here we analyse one of the clearest examples in detail. Figure 10 shows the evolution of Doppler shift, red wing intensity, and line width of the Fe XIX line along the slit. We find that the feature propagating from B to A is composed of two highly redshifted brightenings which occurred with a delay of several minutes. The appearance of these shifted brightenings are the main cause for the initial large line broadenings (see Fig. 10c). The initial feature of the Doppler oscillation at $\mathrm{C}$ is distinctly different from those at $\mathrm{A}$ and $\mathrm{B}$. The initial phase of the oscillation at $C$ is similar to case $E$ found in Sect. 3.2 in that it does not show a highly shifted brightening nor large line broadening. Importantly, we find that the oscillations at $\mathrm{A}$ and $\mathrm{C}$ are associated with intensity fluctuations (Fig. 11). There is a phase shift of a quarter period between the velocity and intensity oscillations after approximately 20:30, indicating that standing slow waves are set up after the first shift pulse for case A. For case $\mathrm{C}$ the standing wave is set up about a quarter period earlier, i.e. right from the first perturbation. Therefore, we exclude propagating waves as an explanation of the phase propagation.

The evolution of the Fe XIX spectra along the slit in the initial phase is shown in Fig. 12a. The shifted brightening at B 

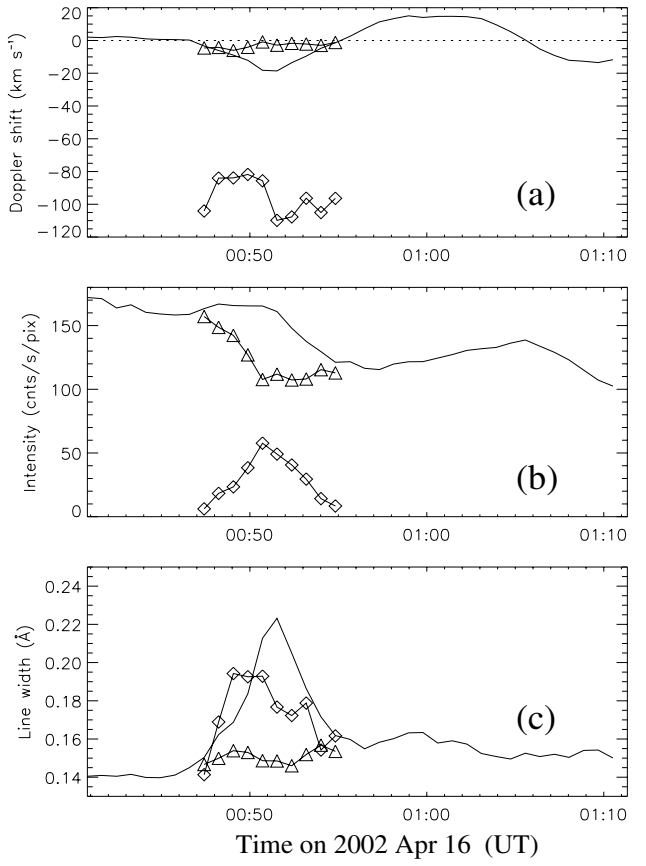

Fig. 9. a) Time profiles of Doppler shifts for cut A, shown in Fig. 5a. The solid line represents the Doppler shifts given by a single Gaussian fit. Triangles and diamonds represent Doppler shifts of two components given by a double-Gaussian fit. b) Corresponding time profiles of line-integrated intensity. c) Corresponding time profiles of line width.

started at 20:22:58, about a minute earlier than that at A. Both brightenings occurred overlaid on the background emission, and thus exhibited the line profiles of two components. We use double-Gaussian fits to measure the Doppler velocity of the highly shifted component. We obtain a maximum shift of $162 \mathrm{~km} \mathrm{~s}^{-1}$ at 20:23:48 for brightening B and of $220 \mathrm{~km} \mathrm{~s}^{-1}$ at 20:25:27 for brightening A. The background component at B is much stronger than at A, because there was a preheating process at $\mathrm{B}$ which happened $10 \mathrm{~min}$ before the onset of the shifted component. The spectral evolution at $\mathrm{C}$ is distinctly different from those at $\mathrm{A}$ and $\mathrm{B}$, with the background emission increasing when the line center position became redshifted. Evolution of the Fe XIX spectra for seven serial cuts along the slit are shown in Fig. 12b, revealing the presence of three different Doppler oscillations along the slit, i.e., frame 1 shows oscillation $\mathrm{C}$, frames 2-4 show oscillation $\mathrm{B}$, and frames 6-7 show oscillation A, with a longer period. Frame 5 shows aspects of both oscillation $\mathrm{A}$ and $\mathrm{B}$.

Various differences revealed in the initial spectra for the oscillations $\mathrm{A}, \mathrm{B}$, and $\mathrm{C}$ indicate the presence of three oscillating loops. We suggest that a sequence of hot plasma ejections occurred at the footpoints on the same side of two neighboring loops A and B. These triggered the standing slow waves. At the same time the pressure disturbance associated with the strong energy release at the footpoint of loop B excited the standing wave in loop $\mathrm{C}$. The phase propagating feature can be explained by the fact that oscillations in loops $\mathrm{A}$ and $\mathrm{C}$ are excited with a time delay with respect to oscillation $\mathrm{B}$, and also due to the

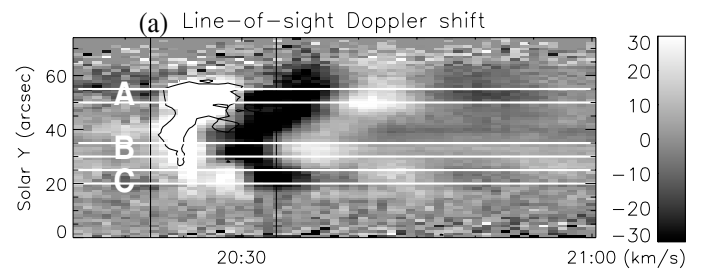

(b) Line-integrated intensity

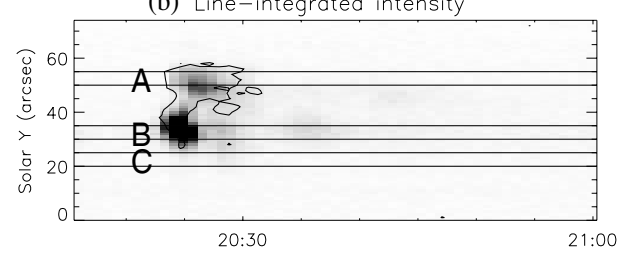

(c) Doppler line width

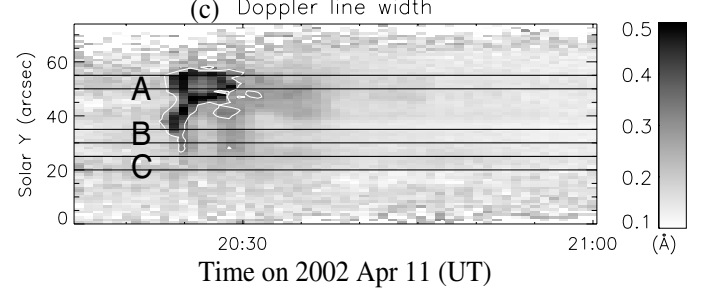

Fig. 10. a) Doppler shift time series of the Fe XIX line at a fixed slit position. The two vertical lines mark an interval of time, within which the spectra along the slit are shown in Fig. 12a. b) Line intensity time series integrated in the red wing from a Doppler shift of $130 \mathrm{~km} \mathrm{~s}^{-1}$ to $250 \mathrm{~km} \mathrm{~s}^{-1}$. c) Line width (Gaussian width) time series. The overlaid contours outline areas of line width above $0.3 \AA$. The labels A, $\mathrm{B}$ and $\mathrm{C}$ mark sections with a width of 6 pixels along the slit that are discussed in the main text.

fact that the oscillation periods at $\mathrm{A}$ and $\mathrm{C}$ are longer than that at B.

\subsection{Summary of main features of the three examples}

1. All three events show the presence of two components in the Fe XIX spectra during the initial phase. One component is almost stationary and the other has a high Doppler shift. Since the intensity of the shifted component increases impulsively and then decays before the oscillation starts, this feature suggests the passage of ejected plasma through the slit.

2. We find intensity fluctuations associated with Doppler shift oscillations in the last two events. The fact that a phase shift of 1/4-period between them appears immediately after the initial Doppler shift pulse suggests that slow mode standing waves are set up quickly in coronal loops (i.e., in about a half period). This result is confirmed in the first event by the fact that after the initial flow is seen to pass from $A$ to $B$, the plasma at the two points along the loop oscillates in phase.

3. We find strong evidence for ejected hot flow along a loop in the first event, which was seen as two brightenings separated by a time lag and located near positions where the SUMER slit intersected a TRACE loop. The estimated transit time of the flow along the loop computed from the Doppler shifts are comparable to the timing of the brightenings. 

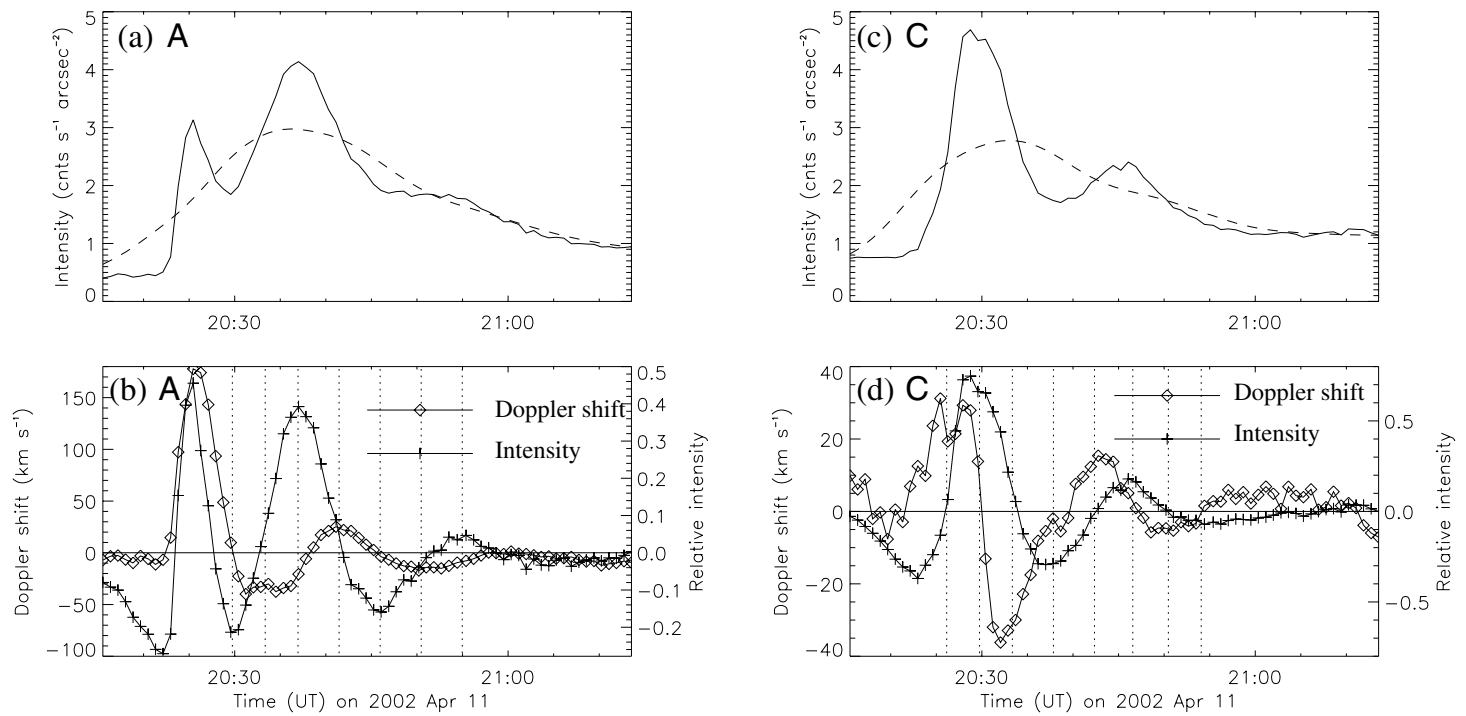

Fig. 11. a) Time profile of intensity (solid line) in the Fe XIX line for cut A along the slit shown in Figs. 10a and 12a. The dashed line is assumed to represent the background trend, which is obtained by a 20-pixel smoothing. b) Doppler and intensity oscillations for cut A. For the intensity curve, a background trend (dashed line in frame a)) has been subtracted, and the variation is normalized relative to the maximum value of the background trend. c)-d) Same as a) and b), but for cut C. In b) and d), the dotted lines are used to display the phase shift between Doppler and intensity oscillations.

(a)
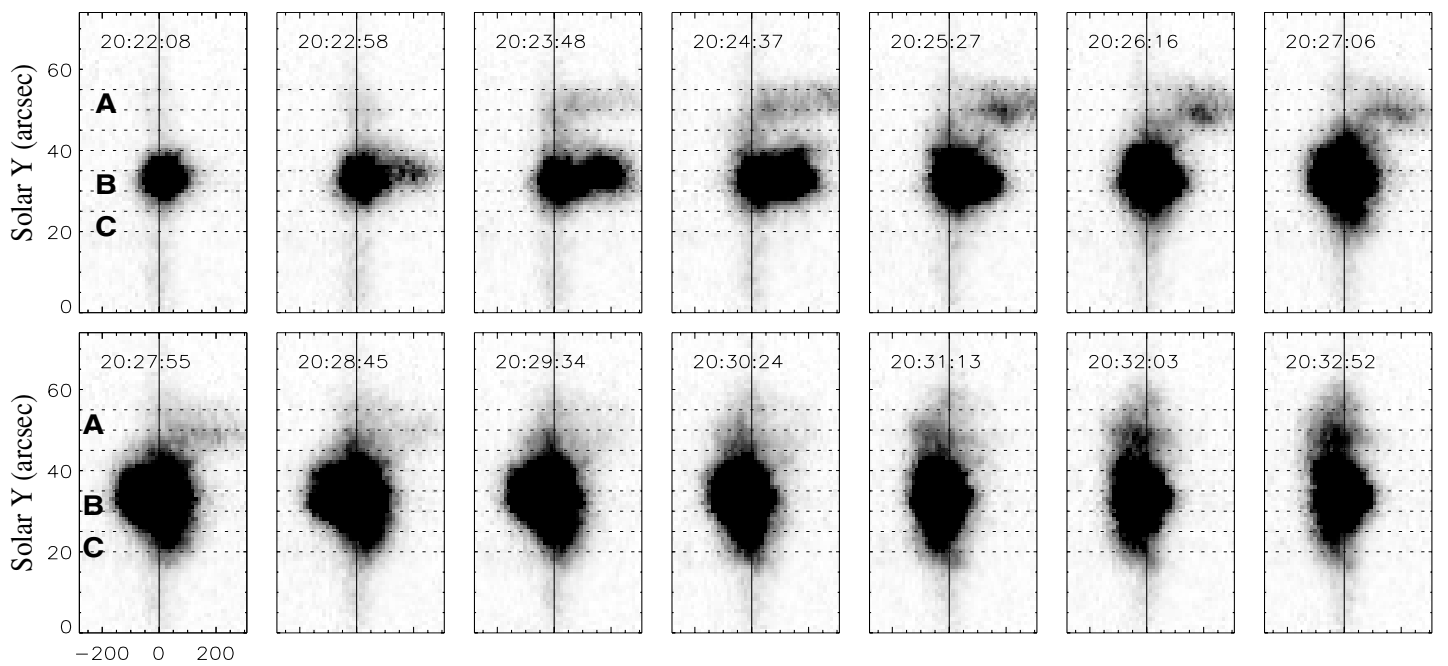

Doppler shift $(\mathrm{km} / \mathrm{s})$

(b)
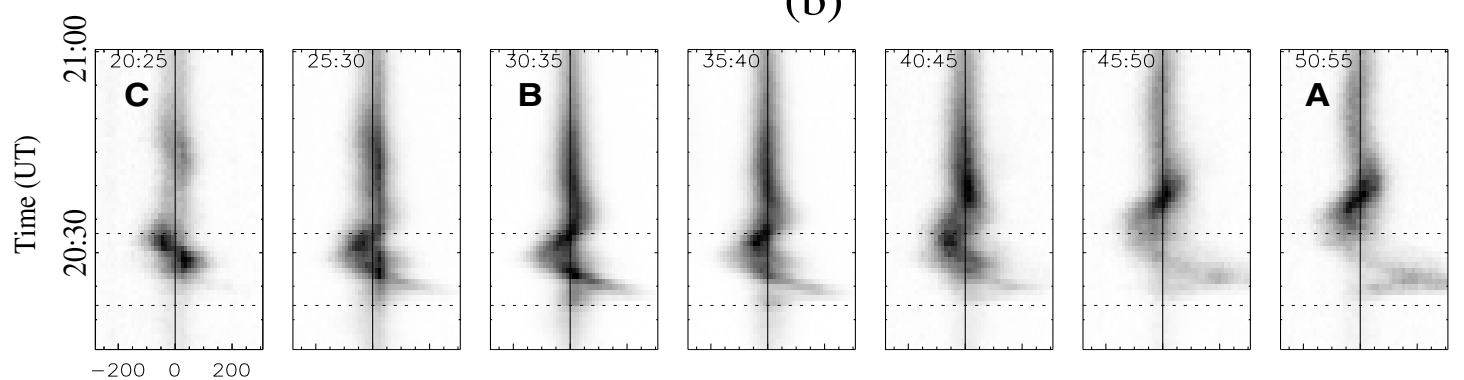

Doppler shift $(\mathrm{km} / \mathrm{s})$

Fig. 12. Evolution of the Fe XIX spectra for the Doppler oscillation event on 11 April 2002. a) Series of spectra along the slit at different times. b) Time series of the average spectra for serial cuts along the slit (marked with the dotted lines in a)). For each frame, the position of its cut is shown at the top. In a), the spectral intensity has the same gray scales for all frames, while in $\mathbf{b}$ ), the intensity is scaled according to the minimum and maximum values for each frame in order to be seen clearly. 
4. Careful analyses reveal that the phase propagation of initial Doppler shifts along the slit for the last two events are produced by several highly shifted brightenings, suggesting that this feature could result from a sequence of plasma ejections occurring in one footpoint of a loop system (consisting of several neighboring loops).

\section{Statistical results}

\subsection{Characteristics of initial spectra}

We re-examine the line profiles of the 54 Doppler oscillations reported in Paper I, and find that they can be classified into four types based on the evolution of their spectral features in the initial phase.

Type 1 - Double components:

There are 26 cases showing the presence of two spectral components at the initial phase (i.e., 4A, 5A, 6C, 6D, 7A, 9C, 11B, $11 \mathrm{C}, 12 \mathrm{E}, 13 \mathrm{~A}, 13 \mathrm{~B}, 14 \mathrm{D}, 15 \mathrm{~A}, 15 \mathrm{~B}, 15 \mathrm{C}, 15 \mathrm{D}, 17 \mathrm{~A}, 18 \mathrm{C}$, 19A, 19B, 20A, 20C, 24A, 26B, 26C, 26D, see Table 1 in Paper I). All are observed in the Fe XIX line, and have background emission near the line center prior to the events. These events commonly start as the appearance of a highly shifted component, overlaid on the pre-existing background component, which shows a weak shift with a small increase of the intensity when the event happens. In 7 cases (e.g. case A in example-3), the intensity of the shifted component quickly increases to become a factor of 2-5 larger than that of the background component, and so the line profile is dominated by a single peak of the shifted component. This peak then decays and is shifted to the line center and oscillates as a single component. This suggests that the initial pulse sets up an oscillation in the "background" gas, although alternative scenarios cannot be ruled out. In 10 cases (e.g. cases A and B in example-1), the highly shifted component decays and merges with the background component, and then is shifted to another wing with a separation from the background component. This suggests that the background emission may be emitted from other undisturbed loops. In 2 cases (e.g. case A in example-2), the background component starts to oscillate after the almost complete decay of the highly shifted component. In 5 cases, the intensity of the shifted component is weaker than the background component, and so its evolution is similar to the type 3 events.

Type 2 - Line center shift:

There are 12 cases of this type (i.e., $2 \mathrm{~B}, 3 \mathrm{C}, 3 \mathrm{D}, 4 \mathrm{~B}, 4 \mathrm{C}, 8 \mathrm{C}$, $11 \mathrm{D}, 14 \mathrm{C}, 16 \mathrm{E}, 16 \mathrm{~F}, 18 \mathrm{D}, 23 \mathrm{~A})$, which are all observed in the Fe XIX line. These events start with a shift of the background component, with a line broadening and strong intensity increase. This component then begins to oscillate.

\section{Type 3 (or type 1 b) - Line wing enhancement:}

There are 7 cases of this type (i.e., 5B, 8B, 9A, 9B, 10A, 18B, 20B), which are all observed in the Fe XIX line. These events start as an intensity enhancement in the wing of the background component, so that the line profile becomes asymmetric. The peak intensity shows little change in 4 out of the 7 cases and an increase by a factor of 2 in the other 3 cases. In most cases, the background component starts the oscillation and shows the increase of the peak intensity when the line wing enhancement decays.

Type 4 (or type 1c) - Single shifted component without the background:

We found 9 examples of this type in our data set (i.e., 1A, 22A, 27A, 27B, 27C observed in the Fe XXI line, and 2A, 21A, $21 \mathrm{~B}, 25 \mathrm{~A}$ in the Fe XIX line). There is no background emission for the cases seen in Fe XXI and negligible emission for these cases seen in Fe XIX prior to the events. These events start with the appearance of a highly shifted component. This component then moves towards the line center with the intensity either increasing or decreasing. It continues to move as part of the oscillation.

By comparison, we find that there is a common feature for types 1, 3 and 4: i.e., that all these events start with the appearance of a highly shifted component, but this component is much weaker compared to the background component in the case of type 3 , while there is no background component in the case of type 4 . In other words, types 3 and 4 can be regarded as two special cases of type 1 . We shall therefore refer to types 3 and 4 as $1 \mathrm{~b}$ and $1 \mathrm{c}$ in the following. For these types, the shifted component has a high Doppler velocity. For example, for the 9 cases of type 4 (1c), the initial maximum velocity has a mean value of $149 \pm 63 \mathrm{~km} \mathrm{~s}^{-1}$. In contrast, for the cases of type 2, the events start with a weak shift of the background component. The average maximum shift is measured to be $55 \pm 35 \mathrm{~km} \mathrm{~s}^{-1}$. Their difference seems to suggest the cases of type 2 may be produced by the compression front of pressure disturbances, while the other three types of cases are produced by ejected hot plasma moving along the loop. Because the oscillation events were detected in loops sampled 50-100 arcsec off the limb of the Sun, the ejected hot plasma could reach the slit position only for those strong events. In this case the emission from the moving plasma overlaid with the undisturbed background emission will produce the spectra showing two components. For the weak events the ejected plasma could have insufficient kinetic energy to reach the loop top, so that only the associated perturbation can propagate and pass through the slit. Hence the plasma that gets disturbed in the loop will produce simple shifted spectra as seen in the cases of type 2. Nevertheless, we note that since the orientations of the loops and therefore the true flow speeds are unknown, we cannot rule out that profiles of type 2 are simply type 1 cases for loops lying in a plane almost perpendicular to the line of sight. This interpretation, however, is not consistent with the fact that the amplitudes of the type 2 oscillations are similar to the type 1 oscillations.

\subsection{Velocity measurements for two-component cases}

For the cases of type 1, we measure the velocity of the highly shifted component by using double-Gaussian fits. Figure 13a shows the statistical distribution of the measured results. We find that the line-of-sight speed of the shifted component lies in the range $78-241 \mathrm{~km} \mathrm{~s}^{-1}$, with a mean value of $155 \pm$ $52 \mathrm{~km} \mathrm{~s}^{-1}$, which is similar to the average value found for the type 1c events (see Table 1). This further supports the identification of the events of type $1 \mathrm{c}$ as a subset of type 1 . For 

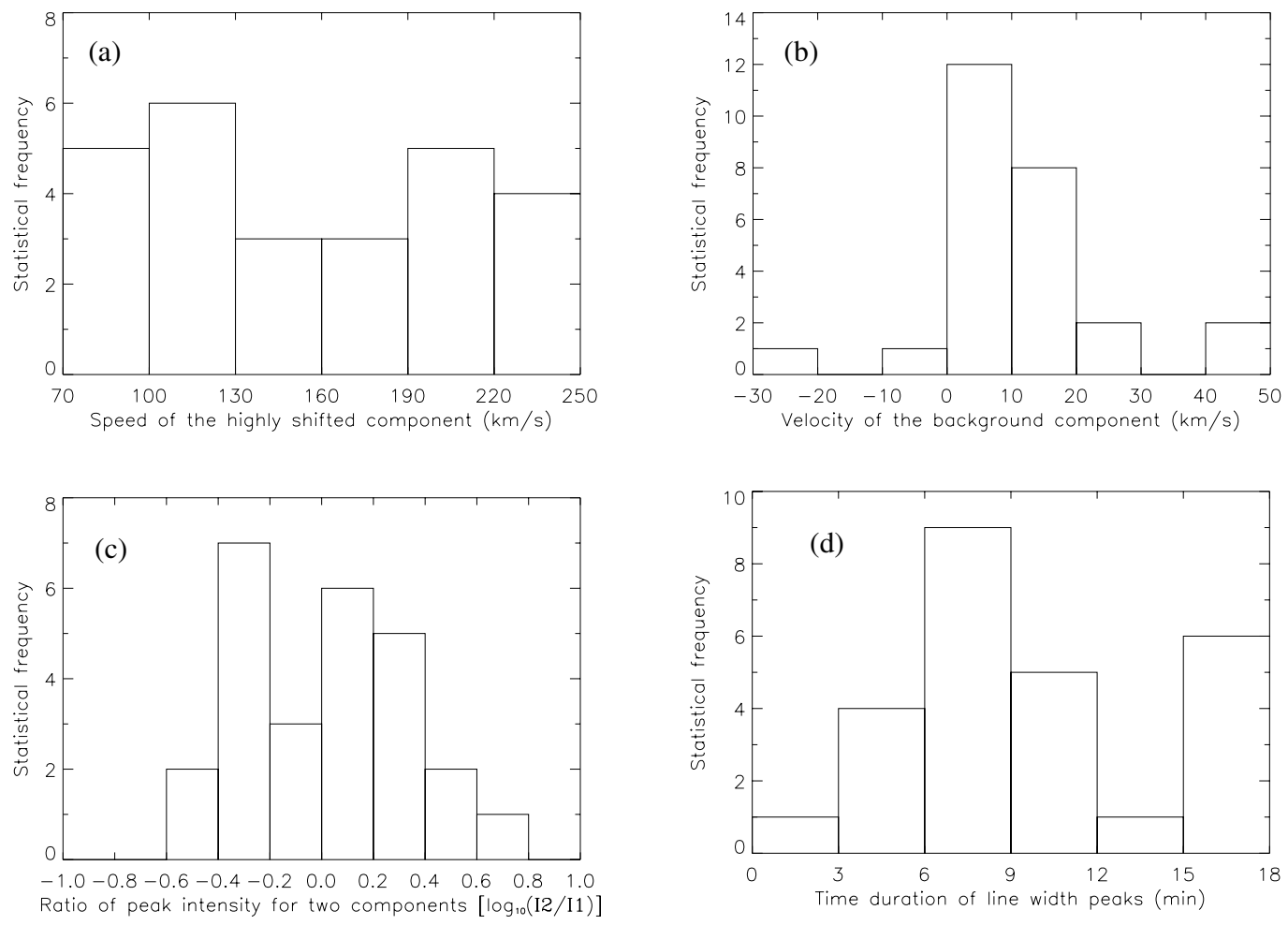

Fig. 13. Distribution of physical parameters for the 26 oscillation cases, with initial line profiles showing two spectral components. a) Maximum (unsigned) line-of-sight speed of the highly shifted component. b) Line-of-sight velocity of the corresponding background component. The sign is positive when the shift has the same sign as of the highly shifted component, negative otherwise. c) Logarithmic ratio of peak intensity of the highly shifted component to that of the background component. d) Duration of the line width peak, where line widths are measured by a single Gaussian fit.

Table 1. Average and range of physical parameters of 54 Doppler-shift oscillations ${ }^{a}$.

\begin{tabular}{|c|c|c|}
\hline Parameters & Average & Range \\
\hline Oscillation period $P$ & $17.9 \pm 5.5 \mathrm{~min}$ & $8.6-32.3 \mathrm{~min}$ \\
\hline Decay time $\tau_{\mathrm{d}}$ & $13.9 \pm 6.7 \mathrm{~min}$ & $3.1-42.3 \mathrm{~min}$ \\
\hline Doppler oscillation amplitude $V_{\mathrm{D}}$ & $62 \pm 57 \mathrm{~km} \mathrm{~s}^{-1}$ & $12-353 \mathrm{~km} \mathrm{~s}^{-1}$ \\
\hline Net flow $V_{0}$ & $3.2 \pm 2.6 \mathrm{~km} \mathrm{~s}^{-1}$ & $0-10 \mathrm{~km} \mathrm{~s}^{-1}$ \\
\hline Velocity of background component ${ }^{b}$ & $8.8 \pm 14.3 \mathrm{~km} \mathrm{~s}^{-1}$ & $-27-46 \mathrm{~km} \mathrm{~s}^{-1}$ \\
\hline Velocity of highly shifted component ${ }^{b}$ & $155 \pm 52 \mathrm{~km} \mathrm{~s}^{-1}$ & $78-241 \mathrm{~km} \mathrm{~s}^{-1}$ \\
\hline Ratio of peak intensity for two comp. ${ }^{b, c} I_{2} / I_{1}$ & $1.37 \pm 1.2$ & $0.34-5.9$ \\
\hline Duration of line width peak ${ }^{b} \Delta T_{\mathrm{w}}$ & $9.4 \pm 4.2 \mathrm{~min}$ & $2.7-17.8 \mathrm{~min}$ \\
\hline Shift of initial pulse for type $1 c^{d}$ & $149 \pm 63 \mathrm{~km} \mathrm{~s}^{-1}$ & $62-234 \mathrm{~km} \mathrm{~s}^{-1}$ \\
\hline
\end{tabular}

${ }^{a}$ The amplitude, damping time and period are obtained by fitting with a damped sine-function to the time profile of Doppler shift oscillations excluding the first peak.

${ }^{b}$ These quantities are measured for the 26 cases of type 1 with initial spectra showing two components. The sign of the velocity for the background component is defined as positive (negative) when its shift has the same (opposite) sign to that of the highly shifted component.

${ }^{c} I_{2} / I_{1}$ is the ratio of peak intensity of the highly shifted component to the background component.

${ }^{d}$ This quantity is measured for the 9 cases of type $1 \mathrm{c}$, with initial spectra showing a single component without the background.

type $1 \mathrm{~b}$ the shifted component is so weak that the results of two-component fits were relatively unreliable and are not discussed further. The velocity of the corresponding background component is in the range $0-46 \mathrm{~km} \mathrm{~s}^{-1}$, with a mean of $9 \pm$ $14 \mathrm{~km} \mathrm{~s}^{-1}$, which is much smaller than that of the highly shifted component. Except for two cases (denoted by negative values in Fig. 13b), the background component is shifted in the same direction as the highly shifted component. We also measure the ratio of peak intensities of these two components for the corresponding line profiles, which is in the range 0.34-5.9 (Fig. 13c). We find that the highly shifted component reaches the maximum shift $\left(V_{\mathrm{m}}\right)$ almost simultaneously with (or a few minutes earlier than) the maximum peak intensity $\left(I_{\mathrm{m}}\right)$. Closer inspection shows that out of the 26 cases, in 10 cases $V_{\mathrm{m}}$ and $I_{\mathrm{m}}$ are simultaneous, in 12 cases $V_{\mathrm{m}}$ is earlier than $I_{\mathrm{m}}$ by $50-170 \mathrm{~s}$, in 3 cases $V_{\mathrm{m}}$ is later than $I_{\mathrm{m}}$ by $50 \mathrm{~s}$ or less (where the minimum cadence is $50 \mathrm{~s}$ ). This result can be explained by a pulse of ejected hot plasma passing through the slit. The duration of 
the pulse of the highly shifted component is difficult to measure because usually only a few spectra with a clear two-component feature are available which allow double-Gaussian fits. Note that the initial strong line broadenings are mainly due to the appearance of the highly shifted component, as seen in the case studies (Sect. 3). Thus we may take the duration of the line width peak as an estimate for the upper limit of the highly shifted component duration (see Fig. 6b). We find that the $F W H M$ of the duration of the line width peak has a mean value of $9.4 \pm 4.2 \mathrm{~min}$ (Fig. 13d), which is about half of the oscillation period. The averages of and the ranges covered by the measured physical parameters are summarized in Table 1.

\subsection{Physical parameters of the oscillations: A reappraisal}

In the case studies, we found that the standing slow waves are set up only after the first peak of the Doppler oscillations dies away. In the 26 two-component cases, the fact that the initial highly shifted component reaches its maximum shift and peak intensity almost simultaneously, also indicates that the initial Doppler peak is not part of the standing wave oscillation. In Paper I, we measured physical parameters of Doppler oscillations such as the amplitude, period and damping time based on the whole time series. Therefore, our measurements could include a bias due to the inclusion of the first peak. Here we remeasure these parameters by using a damped sine-function to fit the Doppler oscillations excluding the first peak. We summarize the newly obtained values in Table 1 . We find that the periods are almost unchanged, the damping time is shorter by $5 \%$, and the amplitude is smaller by $37 \%$ than the average values listed in Paper I. For the scaling of the decay time with the period for 51 cases (excluding 3 dubious cases) we obtain

$\tau_{\mathrm{d}}=0.84_{-0.33}^{+0.56} P^{0.96 \pm 0.18}$

with a correlation coefficient of 0.55 (see Fig. 14a). This result reflects that the damping is slightly stronger than that measured before. Previously, $\tau_{\mathrm{d}}=0.68 P^{1.06 \pm 0.18}$ had been found.

We find that the velocity amplitudes of the Doppler oscillations are almost independent of the maximum shift of the highly shifted component for the cases of type 1 and type 1c (Fig. 14b), while there is a rough linear dependence (with a correlation coefficient of 0.6 ) between the amplitudes for the cases of type 2. This difference also suggests that the initial Doppler peak for the two-component cases (including types 1, $1 \mathrm{~b}$ and 1c) and the line center shifted cases (type 2) could be produced in different ways (see the discussion in Sect. 4.1).

\subsection{Net flows}

We measure the net (background) Doppler flows during the oscillations by fitting an offset damped sine-function (Eq. (1) in Paper I) to the oscillation time profiles excluding the first peak. We find that the net shifts are in the range $0-10 \mathrm{~km} \mathrm{~s}^{-1}$ with a mean of $3.2 \pm 2.6 \mathrm{~km} \mathrm{~s}^{-1}$ (Fig. 15a), which is about a factor of 10 smaller than the minimum Gaussian widths of the Fe XIX line measured after the oscillation events. Figure 15b
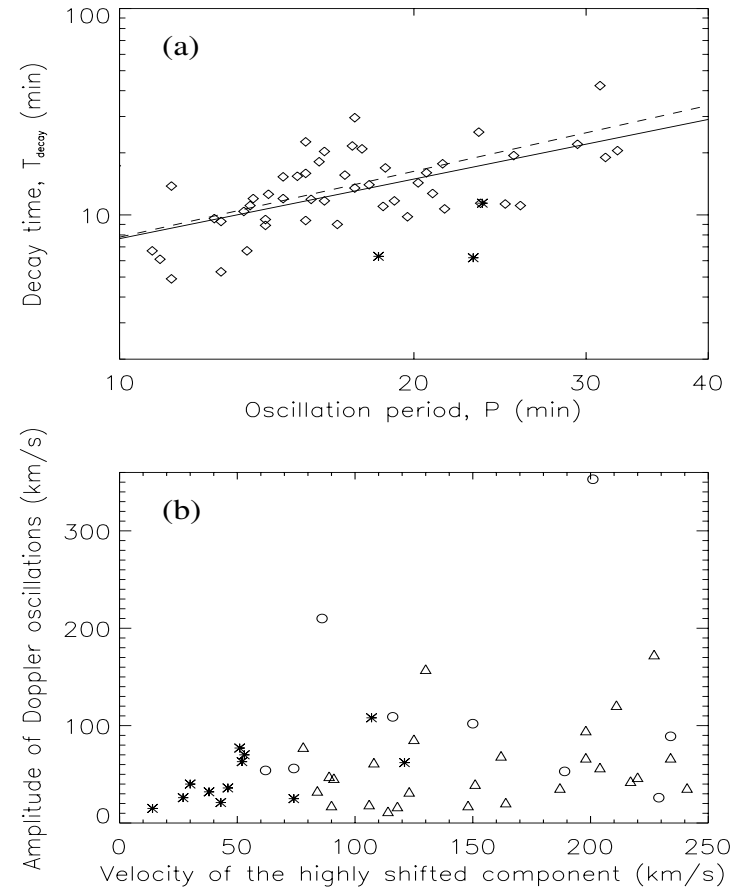

Fig. 14. a) Scaling of the damping time of Doppler oscillations with their period. The solid line is the best fit scaling for 51 oscillations (diamonds), excluding 3 dubious cases (asterisks, i.e. 19A, 21A, and 25A). The dashed line is the best fit scaling for 49 oscillations obtained in Paper I, where the physical parameters are measured by fitting the full time series including the first peak of the oscillations. b) Amplitude of Doppler shift oscillations with respect to the maximum velocity $\left(V_{\mathrm{m}}\right)$ of the highly shifted component for the 26 cases of type 1 with double components (triangles), for the 12 cases of type 2 (asterisk), and for the 9 cases of type 1c (circles), where $V_{\mathrm{m}}$ for the cases of type 2 and type $1 \mathrm{c}$ is the maximum of the first peak of Doppler oscillations. In all frames, the amplitude, damping time and period are obtained by the fitting of Doppler oscillations excluding the first peak.

shows that the distribution of the net line-of-sight flows is roughly symmetric around zero. Here we define the net shift as positive when the signs of the net shift and the initial peak shifts are the same, while it is negative when they are opposite. This result indicates that the net flows are not determined by the initial ejected flows. In addition, by examing the evolution of Doppler oscillations for the 54 cases studied in Paper I, we do not find the presence of any evident background trend. For similar Doppler oscillation events, Feldman et al. (2004) also found that little or no net Doppler flows are present. In this context, they interpreted the strong density increase measured at peak brightness in terms of a compression of the heated coronal plasma. In our study, however, we find that the initial high Doppler shifts are not caused by the oscillation flows, but by the ejected flows from one footpoint of the loop. This initial plasma ejection is likely to contribute significantly to the density increase in the loop.

\section{Excitation by single footpoint heating}

In Paper I we found that the initiation of several oscillation events was associated with the brightening of a footpoint of a coronal loop seen in soft X-ray images recorded by 

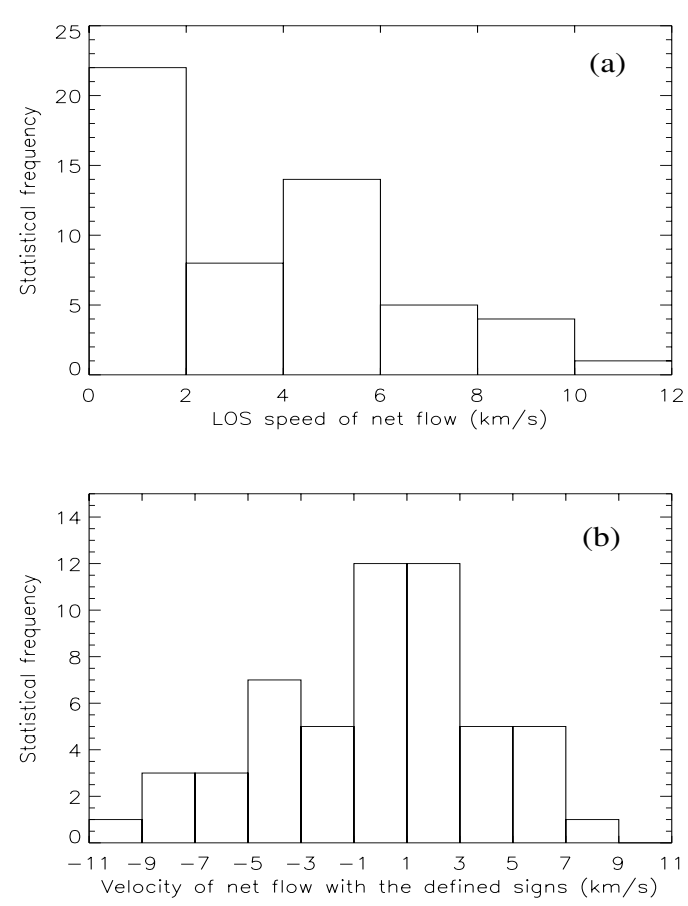

Fig. 15. Distributions of the line-of-sight speed of net Doppler flows for all 54 oscillations. a) Speed of the net flow. b) Same as a) but introducing a sign such that the net flow is positive (negative) when its sign is the same as (opposite to) that of the initial shift.

Yohkoh/SXT. These events suggest that the hot loop oscillations are excited by events such as small flares at a footpoint, i.e., by single footpoint heating. A MHD simulation showed that thermal energy perturbations near the footpoint of a loop can drive both shocks and plasma flows along the loop (Sarro et al. 1999). The initial hot plasma ejections inferred from the two-component feature for many oscillation events are consistent with this picture. These events could be preceded by a shock front or compression disturbance, which travels along the loop and is reflected at the other end. Thus, such a pulse could in principle excite standing slow-mode waves in the loops.

Recent simulation work of Nakariakov et al. (2004) and Tsiklauri et al. (2004) has shown that the 2nd harmonic of a standing slow-mode wave is excited in a flaring loop by both apex (symmetric) heating and single footpoint (asymmetric) heating. Simulations by Selwa et al. (2005) confirm the results of the other simulations for the excitation at the apex, but produce the first standing mode if the excitation takes place further away from the loop top, in contradiction to the result of Tsiklauri et al. (2004). Simulations by Taroyan et al. (2005) support the footpoint excitation of the first standing mode, and find that the oscillations are excited most efficiently when the duration of the heat deposition is equal to the fundamental period of the loop.

To simulate the excitation of slow mode standing waves in hot loops observed by SUMER, consideration of the following properties should be important.

1. The standing slow-mode waves are set up quickly about a half period after the onset of the events.
2. These oscillations are the fundamental mode of slow mode standing waves, suggested by the fact that the oscillation period is roughly equal to twice the loop length divided by the sound speed when soft X-ray images of the hot oscillating loop are available (see Paper I; and Innes \& Wang 2004). No evidence for the 2 nd harmonic has yet been found in our observations. In this the observations tend to support the simulations of Selwa et al. (2005).

3. The loop temperature is initially at about 2-3 MK, which is typical for high-altitude coronal structures seen by SXT (Sterling et al. 1997). In oscillation events the loop plasma is impulsively heated to a temperature of 6-8 MK, and then is gradually cooled back to the initial temperature. The heating of oscillating plasma is indicated by strong brightening at the loop apex seen in the Fe XIX line and a simultaneous dimming in Ca XIII (2.3 MK), and the cooling of hot plasma is clearly indicated by time delay of emission peaks in the lines at lower temperatures (Wang 2004; Feldman et al. 2004). The duration of the heating is about several minutes, and the cooling lasts for several hours.

4. The duration of the brightenings is about several times as long as the oscillation period.

5. Except for strong initial ejected flows lasting for about half a wave period, there is an absence of background flow. This suggests that the observed impulsive ejected flows are not produced by chromospheric evaporation. An alternative is the transient flow accelerated by magnetic reconnection in one flank of the loop.

Another interesting feature shown in the simulation of Tsiklauri et al. (2004) is the phase propagation of the velocity oscillations along the loop in the asymmetric case (see Fig. 4 in their paper), which is very similar to that observed in some events of SUMER Doppler oscillations. We suggest that this propagation feature is due to a sequence of events triggering oscillations in the fine structure of a loop system, but the simulations provide an alternative interpretation in terms of the oscillations of the node in the velocity.

\section{Summary}

In this paper, we have studied the excitation of hot loop oscillations observed with SUMER by a detailed analysis of the evolution of spectral line profiles in the initial phase. We first diagnosed three selected examples in detail. In the first case, we found evidence for hot plasma flowing through the loop from one footpoint towards the other. This flow appears to trigger the loop oscillations. In the other two cases, we found that the standing slow waves are set up quickly after an initial pulse. In all three cases, the events started with the appearance of a highly shifted component in the wing of the pre-existing background component. This highly shifted component reached its maximum shift and peak intensity almost simultaneously, which is consistent with the signature of a pulse of ejected hot plasma, as seen by a slit spectrometer. In two cases, the initial Doppler shifts display a phase propagation along the slit. We found that this behavior could be produced by a sequence of triggers of hot plasma ejection in a loop system. 
We then statistically studied the 54 Doppler shift oscillations in 27 brightening events that had earlier been identified in Paper I. We found that the line profiles for nearly $50 \%$ of the cases are composed of two components in the initial phase. For an additional $30 \%$ of the cases, the initial line profiles are asymmetric or appear as a single highly shifted component. The highly shifted component has a maximum shift on the order of 100-200 $\mathrm{km} \mathrm{s}^{-1}$ and its amplitude does not correlate with the amplitude of the Doppler oscillations measured for the time series excluding the first peak. These observations suggest that the initial Doppler shifts are produced by a pulse of hot plasma. For the rest, i.e. about $20 \%$ of the cases, the oscillations start as a relatively weak shift (below $100 \mathrm{~km} \mathrm{~s}^{-1}$ ) of the background component. Their initial shift has a rough linear correlation with the amplitude of Doppler oscillations. We suggest that the initial Doppler shifts for these cases could be produced by a propagating disturbance, although we cannot completely rule out a hot plasma pulse seen in the direction almost perpendicular to its propagation.

We re-measured the physical parameters of 54 Doppler shift oscillations excluding the first Doppler peak when fitting the time profiles, since the profiles during that period are generally not well reproduced by a single Gaussian. We found that the period is almost unchanged compared to the previous results, the damping time is slightly shorter, and the amplitude is reduced by $37 \%$. We also found that the velocity of net (background) flows during the oscillations is nearly zero. For most of the cases, the time series of Doppler shifts did not show any background trend.

The signature of strong ejected hot flows inferred in most cases suggests that the excitation of standing slow mode waves in the coronal loops is by a pulse at or near one footpoint and not by chromospheric evaporation. Such an excitation model is also supported by the fact that the initiation of hot loop oscillations has been seen to be associated with X-ray brightening at one footpoint.

Acknowledgements. SUMER is financially supported by DLR, CNES, NASA and the ESA PRODEX programme (Swiss contribution). SOHO is a project of international co-operation between ESA and NASA.
Error note: The time marks shown in Fig. B.5 of Paper I are wrong, the SUMER observation started at 11:10 UT, not at 11:00 UT, as marked.

\section{References}

Aschwanden, M. J. 2003, NATO Advanced Research Workshop: Turbulence, Waves and Instabilities in the Solar Plasma, ed. R. Erdélyi, et al., 124, 387

Feldman, U., Dammasch, I., Landi, E., \& Doschek, A. 2004, ApJ, 609, 439

Innes, D. E., \& Wang, T. J. 2004, In Proc. 15th SOHO Workshop: Coronal Heating, ed. R. W. Walsh et al. (Noordwijk: ESA), ESA SP-575, 553

Nakariakov, V. M., \& Ofman, L. 2001, A\&A, 372, L53

Nakariakov, V. M., Tsiklauri, D., Kelly, A., Arber, T. D., \& Aschwanden, M. J. 2004, A\&A, 414, L25

Ofman, L., \& Wang, T. J. 2002, ApJ, 580, L85

Roberts, B., Edwin, P. M., \& Benz, A. O. 1984, ApJ, 279, 857

Roberts, B. 2000, Sol. Phys., 193, 139

Roberts, B., \& Nakariakov, V. M. 2003, NATO Advanced Research Workshop: Turbulence, Waves and Instabilities in the Solar Plasma, ed. R. Erdélyi et al., 124, 167

Sarro, L. M., Erdélyi, R., Doyle, J. G., \& Pérez, M. E. 1999, A\&A, 351,721

Selwa, M., Murawski, K., \& Solanki, S. K. 2005, A\&A, in press

Sterling, A. C., Hudson, H. S., \& Watanabe, T. 1997, ApJ, 479, L149

Taroyan, Y., Erdélyi, R., Doyle, J. G., \& Bradshaw, S. J. 2005, A\&A, submitted

Tsiklauri, D., Nakariakov, V. M., Arber, T. D., \& Aschwanden, M. J. 2004, A\&A, 422, 351

Wang, T. J. 2004, In Proc. of SOHO 13, Waves, Oscillations and Small-Scale Transient Events in the Solar Atmosphere: A Joint View from SOHO and TRACE, ed. H. Lacoste, ESA SP-547, 417

Wang, T. J., \& Solanki, S. K. 2004, A\&A, 421, L33

Wang, T. J., Solanki, S. K., Curdt, W., Innes, D. E., \& Dammasch, I. E. 2002, ApJ, 574, L101

Wang, T. J., Solanki, S. K., Innes, D. E., Curdt, W., \& Marsch, E. 2003a, A\&A, 402, L17

Wang, T. J., Solanki, S. K., Curdt, W., et al. 2003b, A\&A, 406, 1105 (Paper I) 